\title{
Assessment of Glycosaminoglycan Content of Lumbar Intervertebral Discs in Patients with Radiculopathy
}

\author{
Sophie Heüveldop ${ }^{1 *}$, Florian Fichter ${ }^{1 *}$, Anja Müller-Lutz ${ }^{1}$, Markus Konieczny², Markus Eichner ${ }^{1}$, \\ Hans-Jörg Wittsack ${ }^{1}$, Christoph Schleich ${ }^{1 \#}$
}

${ }^{1}$ Department of Diagnostic and Interventional Radiology, Medical Faculty, University of Düsseldorf, Düsseldorf, Germany

${ }^{2}$ Department of Orthopedics, Medical Faculty, University of Düsseldorf, Düsseldorf, Germany

Email: "christoph.schleich@med.uni-duesseldorf.de

How to cite this paper: Heüveldop, S., Fichter, F., Müller-Lutz, A., Konieczny, M., Eichner, M., Wittsack, H.-J. and Schleich, C. (2019) Assessment of Glycosaminoglycan Content of Lumbar Intervertebral Discs in Patients with Radiculopathy. International Journal of Clinical Medicine, 10, 259-269.

https://doi.org/10.4236/ijcm.2019.104020

Received: February 24, 2019

Accepted: April 1, 2019

Published: April 4, 2019

Copyright $\odot 2019$ by author(s) and Scientific Research Publishing Inc. This work is licensed under the Creative Commons Attribution International License (CC BY 4.0).

http://creativecommons.org/licenses/by/4.0/

\section{Open Access}

\begin{abstract}
Objective: To assess glycosaminoglycan (GAG) content of lumbar intervertebral discs (IVDs) in patients with radiculopathy compared with healthy volunteers with glycosaminoglycan chemical exchange saturation transfer (gagCEST). Methods: The lumbar spines of 15 patients with radiculopathy (9 women, 6 men; mean age 45 years; range: 19 - 80 years) and 13 healthy controls (10 women, 3 men; mean age 29 years; range: 19 - 38 years) without lumbar back pain or previous spine surgery were examined at a 3 Tesla (T) magnetic resonance imaging (MRI) scanner in this prospective study. The MRI protocol included standard morphological, sagittal, and transverse T2-weighted (T2w) images of the five lumbar IVDs (L1-S1) to assess Pfirrmann score and to detect disc disorders according to the Combined Task Force classification. To analyze biochemically the lumbar IVDs, a gagCEST sequence was applied to measure the GAG content of the nucleus pulposus (NP) and annulus fibrosus (AF). Results. Patients with radiculopathy indicated significantly lower gagCEST values in NP than healthy volunteers $(2.82 \% \pm 3.12 \%$ vs. $4.09 \% \pm 2.25 \%, P=0.017)$. The GAG content of AF showed no significant difference between volunteers and patients $(2.66 \% \pm$ $2.01 \%$ vs. $1.92 \% \pm 2.56 \% ; P=0.175)$. Conclusions. Patients with radiculopathy presented with lower GAG values than healthy volunteers in NP, indicating an association between pain and IVD degeneration. gagCEST of lumbar IVDs is a powerful, non-invasive tool to investigate early disc degeneration, which we could demonstrate in the NP in our study collective.
\end{abstract}

\section{Keywords}

Glycosaminoglycan Chemical Exchange Saturation Transfer, Radiculopathy, Intervertebral Disc, Lumbar Spine, Early Degeneration 


\section{Introduction}

Low back pain (LBP) is a common disease in the industrialized world with a high lifetime prevalence [1]. It is one of the leading causes of disability and imposes a high socio-economic burden [2]. Intervertebral disc (IVD) degeneration is one of the recognized causes of lower back pain [3]. IVDs consist of an outer annulus fibrosus (AF) and an inner nucleus pulposus (NP). Large proteoglycan molecules with numerous negatively charged glycosaminoglycan (GAG) side chains are a major component of IVDs, especially of the NP, which lead to a high osmotic pressure within the disc providing resistance to compressive loading [2] [4].

Magnetic resonance imaging (MRI) is well established in the assessment of IVD degeneration [5]. On T2-weighted (T2w) MR images, normal, non-degenerated intervertebral discs show a bright signal from the nucleus pulposus and inner fibers of the annulus due to a high amount of water [3] [5] [6]. Degenerative disc alterations can be visualized by a decrease in the water content on T2w images and be morphologically graded according to the Pfirrmann classification system [7]. Due to the degenerative process, the NP loses its translucency and becomes more difficult to distinguish from the surrounding AF [5]. Besides the Pfirrmann classification, IVDs can be graded according to the Combined Task Force (CTF) classification in normal appearance, protrusion, and extrusion of IVDs [8] [9].

Several biochemical MRI techniques have been used to assess and quantify extracellular matrix components of fibrous and hyaline cartilage, such as delayed gadolinium-enhanced MRI of cartilage (dGEMRIC), sodium MRI and T1 rho mapping to visualize the GAG content, and $\mathrm{T} 2 / \mathrm{T}^{\star}$ mapping to visualize collagen structure [10] [11] [12] [13] [14]. One relatively new and promising technique is glycosaminoglycan chemical exchange saturation transfer (gagCEST) [15]. Without the application of a contrast agent or dedicated additional MRI hardware, CEST imaging allows the determination of GAG content in IVDs [16] [17]. gagCEST of the lumbar spine is possible at a magnetic field strength of $3 \mathrm{~T}$. For gagCEST imaging, several images are acquired with presaturation pulses at different offset frequencies around the bulk water resonance and one reference image without saturation. The residual signal normalized to the reference image as a function of the offset frequencies ( $\mathrm{z}$-spectrum) can be utilized to determine and quantify the CEST effect according to magnetization transfer asymmetry ratio (MTRasym) values with respect to the water resonance due to the $\mathrm{OH}$ protons of GAG appearing in a frequency range of 0.9 to $1.9 \mathrm{ppm}$ from the water resonance. The magnitude of the measured MTRasym values correlates directly with the underlying concentration of GAG [18] [19].

Haneder et al. have already shown a significant GAG loss in correlation with degenerative changes of IVDs in people with lower back pain [20]. Schleich et al. showed the same trend in a healthy collective [16]. The aim of our study was to compare the glycosaminoglycan content of the lumbar IVDs in patients with ra- 
diculopathy with that of a healthy collective.

\section{Materials and Methods}

\subsection{Hypothesis}

Our hypothesis was that the gagCEST effect is lower in patients suffering from pain due to radiculopathy compared with healthy volunteers.

\subsection{Study Population}

The study was approved by the local ethics committee. Written informed consent was obtained from all volunteers for this prospective study. Fifteen volunteers with radiculopathy ( 9 women, 6 men; mean age: 45 years; range: $19-80$ years) and 13 healthy volunteers (10 women, 3 men; mean age: 29 years; range: 19 - 38 years) without specific, subacute, and chronic low back pain or previous surgery of the lumbar spine were prospectively enrolled in this study. The diagnosis of radiculopathy originated from the doctor's letter or a clinical examination in our spine ambulance.

\subsection{MR Hardware and Sequence Protocol}

The lumbar spine of all participants was examined in supine position using a clinical whole-body 3T MR system (Magnetom Trio, A Tim System, Siemens Healthcare, Erlangen, Germany). Signal reception was performed using four channel body matrix coils and a 24-channel spine matrix coil. Our MR sequence protocol included a localizer and a $\mathrm{T} 2 \mathrm{w}$ sequence in the sagittal and transverse orientations. Parameters of the sagittal T2w turbo spin echo sequence were as follows: field of view $=300 \times 300 \mathrm{~mm}^{2}$, basic resolution of $256 \times 256$, slice thickness $=3 \mathrm{~mm}$, in-plane resolution $=1.2 \times 1.2 \mathrm{~mm}$, TR/TE $=3100 / 105 \mathrm{~ms}$, number of slices $=15$, flip angle $=160^{\circ}$, two signal averages, number of echoes per slice $=17$ and an acquisition time of 3 minutes and 39 seconds. The parameters of the transversal $\mathrm{T} 2 \mathrm{w}$ turbo spin echo sequence were as follows: field of view $=240 \times 240 \mathrm{~mm}^{2}$, basic resolution of $384 \times 307$, slice thickness $=3 \mathrm{~mm}$, in-plane resolution $=0.8 \times 0.6 \mathrm{~mm}, \mathrm{TR} / \mathrm{TE}=4000 / 113 \mathrm{~ms}$, number of slices $=$ 25 , flip angle $=140^{\circ}$, one signal average, number of echoes per slice $=26$, and an acquisition time of 3 minutes and 38 seconds.

Biochemical imaging was performed using a prototype gagCEST and water saturation shift referencing (WASSR) sequences. CEST and WASSR sequences were composed of a presaturation module and a segmented 2D RF-spoiled gradient echo module. Detailed sequence parameters were given in Table 1.

CEST and WASSR images were motion-corrected using a diffeomorphic image registration approach incorporated in the prototype software fMRLung (Siemens Healthcare, Erlangen, Germany) [21]. The following data analysis was performed using in-house developed MATLAB software (The Mathworks, Inc., Natick, MA, R2012b). A reduction of image noise was performed using an in-plane $3 \times 3$ Gaussian filter. B0 field inhomogeneities were corrected using the 
Table 1. Detailed sequence parameters of the gagCEST and WASSR sequence.

\begin{tabular}{|c|c|c|c|}
\hline & & WASSR & CEST \\
\hline \multicolumn{4}{|c|}{ 2D RF-spoiled GRE module } \\
\hline $\mathrm{T}_{\mathrm{E}} / \mathrm{T}_{\mathrm{R}}$ & {$[\mathrm{ms}] /[\mathrm{ms}]$} & $5.56 / 575$ & $3.01 / 1590$ \\
\hline In-plane resolution & {$\left[\mathrm{mm}^{2}\right]$} & $0.8 \times 0.8$ & $0.8 \times 0.8$ \\
\hline Basic resolution & & $256 \times 256$ & $256 \times 256$ \\
\hline Slice thickness & {$[\mathrm{mm}]$} & 5 & 5 \\
\hline Flip angle & {$\left[{ }^{\circ}\right]$} & 12 & 12 \\
\hline Field of view & {$\left[\mathrm{mm}^{2}\right]$} & $150 \times 150$ & $150 \times 150$ \\
\hline Duration & {$[\min : \mathrm{sec}]$} & 10:40 & $17: 36$ \\
\hline NEX (number of excitations) & & 2 & 2 \\
\hline \multicolumn{4}{|c|}{ Presaturation module } \\
\hline Number of measured frequency offsets & & 42 & 26 \\
\hline Maximum frequency offset $\Delta \omega_{\max }$ & {$[\mathrm{ppm}]$} & 1 & 4 \\
\hline B1-CWAE & {$[\mu \mathrm{T}]$} & 1.5 & 0.3 \\
\hline Number of CEST presaturation pulses & & 1 & 8 \\
\hline PD/IPD & {$[\mathrm{ms}] /[\mathrm{ms}]$} & $100 / 6$ & $100 / 100$ \\
\hline
\end{tabular}

WASSR maximum symmetry algorithm [22]. The offset-corrected CESTcurves divided by the signal without CEST presaturation $\mathrm{S} 0$ are defined as $\mathrm{z}$-spectrum $\mathrm{Z}(\Omega)$. The magnetization transfer asymmetry was defined as $\operatorname{MTR} \operatorname{Rasym}(\mathrm{D} \Omega)=$ $\mathrm{Z}(\mathrm{D} \Omega) \mathrm{Z}(\mathrm{D} \Omega)$, where $\mathrm{D} \Omega$ is the specified frequency shift difference. Evaluation of the gagCEST effect was determined using the MTRasym value in the frequency range from 0.9 to $1.9 \mathrm{ppm}$, which comprises the chemical exchange resonances of GAG hydroxyl protons [15].

\subsection{Data Analysis}

All lumbar IVDs (L1-S1; a total of 140 IVDs) could be imaged successfully without any dropouts. One radiologist with 6 years of experience in musculoskeletal radiology scored all lumbar intervertebral discs according to the Pfirrmann scoring system [7]. The scoring system is based on a five-step grading scale with grade 1 and 2 for non-degenerative discs and grade 3 - 5 for degenerative IVDs according to the nucleus signal intensity, the nucleus structure, the distinction between the nucleus pulposus (AP) and the annulus fibrosus (AF), and the disc height in midsagittal T2w images. According to the CTF classification, the same radiologist scored the IVDs into normal appearing discs and IVDs with protrusion or extrusion [9]. For both analyses, the radiologist was blinded to gagCEST values.

A region-of-interest (ROI) analysis was performed for MTRasym evaluation of the NP and AF to identify the gagCEST effect. All ROIs were selected by a self-acting image processing algorithm that detected the lumbar IVDs automati- 
cally. This lumbar IVD segmentation was performed usingin-house developed MATLAB software. The disc segmentation was based on Bayes classification to divide bone and ligament from disc tissue of the lumbar spine. The segmentation area comprised the lumbar spine. According to the different tissue signal intensity of non-saturated and saturated images, the segmentation tool could distinguish IVDs from the other tissues of the lumbar spine by learning on several training objects before data analysis. The defined ROIs were divided into NP (the innermost $60 \%$ of the IVD) and AF (the remaining region of the IVD). Every automatically positioned ROI was visually checked by one radiologist with 6 years of experience in IVD segmentation. None of the ROIs were manually repositioned.

\subsection{Statistical Analysis}

SPSS (Version 22; SPSS; Chicago, IL) was used for statistical analysis. The mean, confidence intervals for the mean values, median, and standard deviations for the NP and AF were calculated as descriptive statistics. The Lilliefors test was used to verify the normal distribution. Because of the non-normally distributed data, we used Mann-Whitney U tests to compare gagCEST effects in NP and AF for patients and control groups. Results were considered statistically significant at $P<0.05$.

\section{Results}

All completed measurements were technically successful. A total of 140 IVDs (L1-S1) of 15 patients with radiculopathy and 13 healthy volunteers were analyzed. Morphologically, 22 IVDs were scored Pfirrmann grade 1, 71 lumbar discs were scored Pfirrmann grade 2, 28 IVDs were graded Pfirrmann score 3, 18 discs were graded Pfirrmann grade 4, and 1 IVDwas scored Pfirrmann grade 5. Descriptive data are summarized in Table 2.

In non-degenerated (Pfirrmann grade 1-2) discs, significantly higher gagCEST values were found in the NP than in the $\operatorname{AF}(P=0.006)$. In degenerated discs (Pfirrmann grade 3-5), no significant difference between the NP and AF could be detected $(P=0.71)$. We could demonstrate significantly lower gagCEST values in degenerated IVDs than in non-degenerated discs in the NP $(2.18 \% \pm$ $3.57 \%$ vs. $3.99 \% \pm 2.1 \%, P=0.001)$. For the AF, we found no significant difference between degenerated and non-degenerated IVDs. Lumbar discs without protrusion or extrusion showed significantly different gagCEST effects between the NP and AF $(P<0.0001)$. IVDs with protrusion or extrusion revealed no significant difference between the NP and $\operatorname{AF}$ ( $P=0.0923 ; P=0.535$, respectively).

Patients with radiculopathy presented significantly lower gagCEST values in the NP compared withealthy controls $(2.82 \% \pm 3.12 \%$ vs. $4.09 \% \pm 2.25 \%, P=$ 0.017) (Figure 1, Figure 2). In the AF, no significant difference between patients and controls were found $(P=0.175)$. 
Table 2. Descriptive data. Mean, standard deviation (Std), median, minimum, maximum, $95 \%$ confidence interval with lower and upper limit.

\begin{tabular}{|c|c|c|c|c|c|c|c|}
\hline & Mean & Std & Median & Min & $\operatorname{Max}$ & $\begin{array}{c}\mathrm{CI} \\
\text { [lower limit] }\end{array}$ & $\begin{array}{c}\mathrm{CI} \\
\text { [upper limit] }\end{array}$ \\
\hline AF total & 2.27 & 2.35 & 2.44 & -7.78 & 8.09 & 1.8811 & 2.6597 \\
\hline NP total & 3.42 & 2.82 & 3.67 & -10.21 & 12.11 & 2.9486 & 3.8827 \\
\hline AF Pfirr 1 & 1.87 & 1.96 & 1.54 & -0.61 & 5.67 & 1.0500 & 2.6872 \\
\hline NP Pfirr 1 & 3.53 & 1.89 & 3.78 & 0.58 & 7.11 & 2.7429 & 4.3239 \\
\hline AF Pfirr 2 & 2.97 & 1.92 & 3.07 & -1.60 & 8.09 & 2.5300 & 3.4131 \\
\hline NP Pfirr 2 & 4.14 & 2.28 & 4.14 & -0.86 & 12.11 & 3.6169 & 4.6611 \\
\hline AF Pfirr 3 & 1.93 & 1.79 & 2.02 & -2.17 & 6.29 & 1.2370 & 2.6146 \\
\hline NP Pfirr 3 & 2.93 & 2.18 & 2.75 & -1.05 & 8.42 & 2.0985 & 3.7713 \\
\hline AF Pfirr 4 & 0.25 & 3.77 & 1.01 & -7.78 & 5.22 & -1.5388 & 2.0484 \\
\hline NP Pfirr 4 & 0.88 & 4.87 & 2.43 & -10.21 & 6.51 & -1.4414 & 3.1919 \\
\hline AF Pfirr 5 & 3.09 & 0 & 3.09 & 3.09 & 3.09 & 3.0943 & 3.0943 \\
\hline NP Pfirr 5 & 5.03 & 0 & 5.03 & 5.03 & 5.03 & 5.0314 & 5.0314 \\
\hline AF Pfirr $1+2$ & 2.72 & 1.98 & 2.75 & -1.60 & 8.09 & 2.3183 & 3.1139 \\
\hline NP Pfirr $1+2$ & 4.00 & 2.20 & 3.99 & -0.86 & 12.11 & 3.5567 & 4.4409 \\
\hline AF Pfirr 3-5 & 1.31 & 2.81 & 1.78 & -7.78 & 6.29 & 0.4758 & 2.1377 \\
\hline NP Pfirr 3-5 & 2.19 & 3.60 & 2,67 & -10.21 & 8.42 & 1.1300 & 3.2438 \\
\hline AF BSV 1 & 2.27 & 2.29 & 2.38 & -7.78 & 8.09 & 1.8601 & 2.6797 \\
\hline NP BSV 1 & 3.44 & 2.69 & 3.65 & -10.21 & 12.11 & 2.9553 & 3.9196 \\
\hline AF BSV 2 & 2.62 & 1.73 & 2.61 & 0.52 & 6.29 & 1.6396 & 3.5952 \\
\hline NP BSV 2 & 4.04 & 2.20 & 4.24 & 0.26 & 8.42 & 2.7948 & 5.2803 \\
\hline AF BSV 3 & 1.68 & 4.22 & 3.09 & -7.03 & 5.22 & -1.4480 & 4.7998 \\
\hline NP BSV 3 & 2.17 & 5.33 & 4.28 & -9.16 & 6.51 & -1.7833 & 6.1138 \\
\hline AF BSV $2+3$ & 2.27 & 2.82 & 2.66 & -7.03 & 6.29 & 1.0011 & 3.5400 \\
\hline NP BSV $2+3$ & 3.35 & 3.64 & 4.28 & -9.16 & 8.42 & 1.7092 & 4.9863 \\
\hline AF controls & 2.66 & 2.01 & 2.52 & -1.60 & 8.09 & 2.1734 & 3.1540 \\
\hline NP controls & 4.09 & 2.25 & 4.01 & -0.73 & 12.11 & 3.5433 & 4.6396 \\
\hline AF radiculopathy & 1.92 & 2.56 & 2.28 & -7.78 & 8.06 & 1.3480 & 2.5111 \\
\hline NP radiculopathy & 2.82 & 3.12 & 3.38 & -10.21 & 9.19 & 2.1218 & 3.5380 \\
\hline
\end{tabular}

\section{Discussion}

Biochemical alterations of lumbar IVDs are present before morphological changes of the intervertebral disc appear [16] [19] [20]. GAG is one of the major components of the extracellular matrix of IVDs [5]. The loss of tissue water content of the IVD due to a depletion of GAGs plays a central role in these degenerative processes, at first in the NP [2] [4]. 

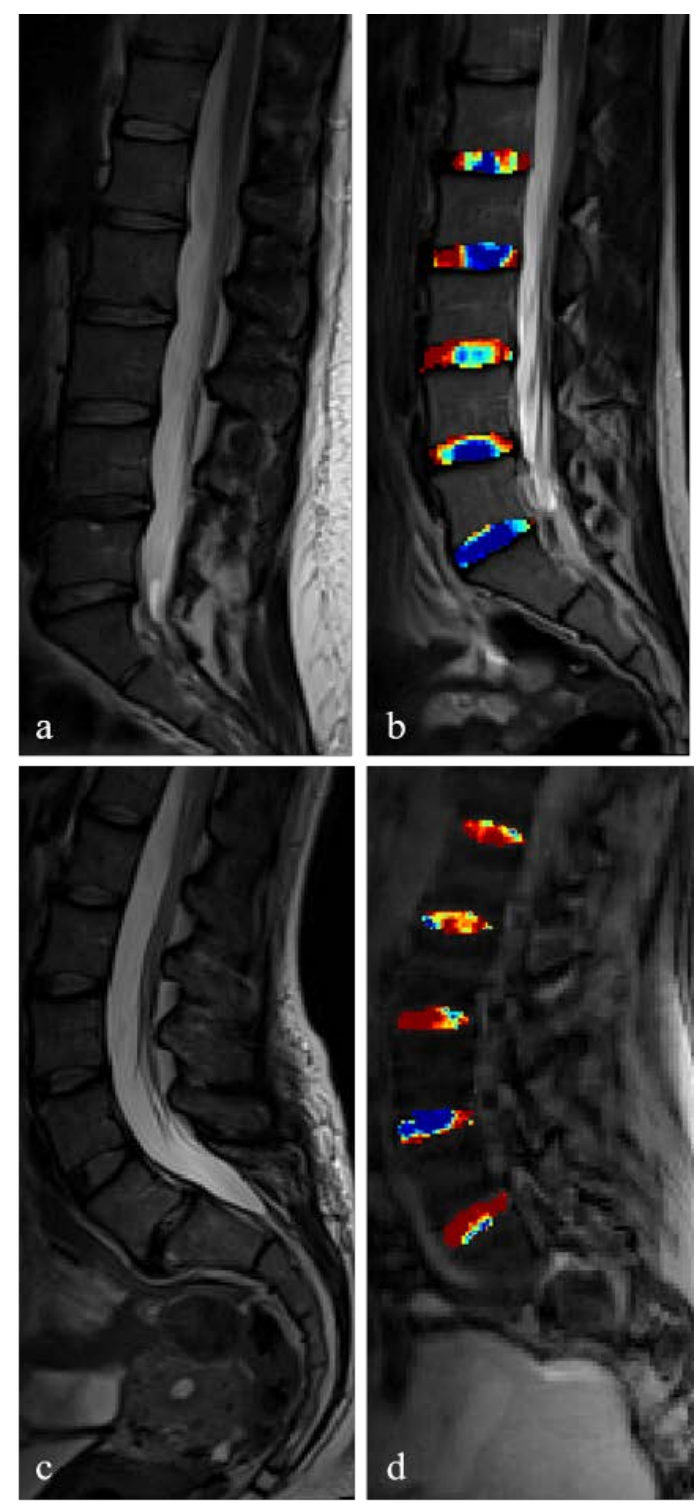

Figure 1. T2-weighted images (image (a), (c)) and color-coded gagCEST maps (image (b), (d)) of the lumbar spine (L1-S1). Morphological images of a healthy control (image (a)) and a patient suffering from radiculopathy (image (c)) revealed almost the same Pfirrmann grading. Biochemical images illustrated lower gagCEST values of patients with radiculopathy compared with healthy controls (image (b), (d)). Especially the motion segment of the affected nerve root (L5/S1) showed the lowest GAG values in this patient (image (d)).

Our results illustrated significantly lower gagCEST values in patients suffering from radiculopathy compared with healthy controls in the NP. For the AF, no significant difference was revealed. In the literature, disc degeneration is considered as one cause of low back pain (LBP) [23]. Additionally, a strong association between disc degeneration and pain has been shown [24] [25]. In accordance with our work, the first degenerative changes could be found in the NP [5] 

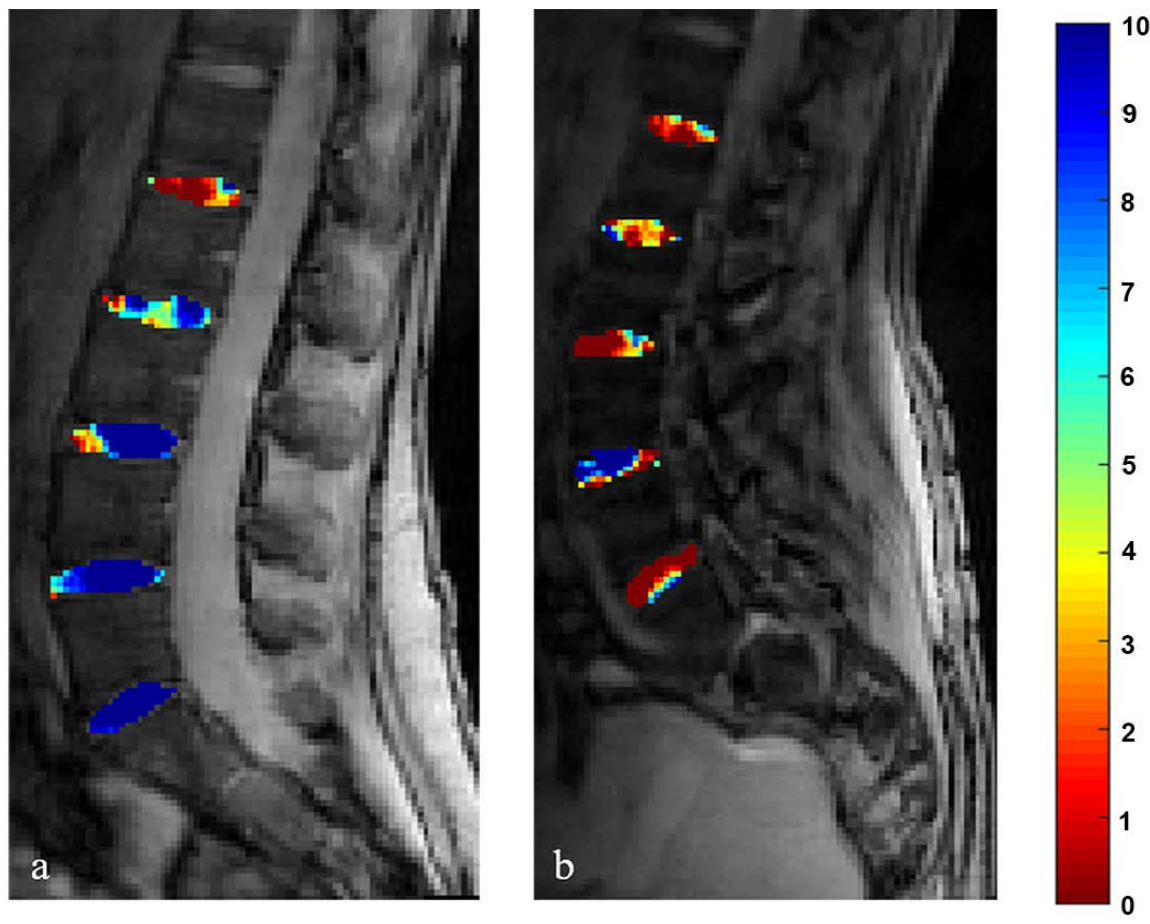

Figure 2. Color-coded gagCEST map with low GAG content in red and high GAG content in blue of the lumbar spine (L1-S1). In this example, a patient suffering from radiculopathy of the segment L5/S1 (image (b)) displayed a significantly lower gagCEST effect compared with a healthy control (image (a)). The low gagCEST effect of segment L1/L2 of the control participant was explainable by motion artifacts of the diaphragm.

[20]. In the AF, degenerative changes are much more difficult to demonstrate due to physiologically lower GAG values in the AF compared with the NP [20]. We found lower GAG values in degenerated IVDs (Pfirrmann grade 3 - 5) compared with healthy discs (Pfirrmann grade 1 and 2) in our data sets. In addition, degenerated discs showed a loss of GAGs in the NP and an adjustment of the AF GAG content in contrast to non-degenerated IVDs, which revealed a significantly higher GAG content of the NP compared with the AF. These findings agree with recent literature demonstrating that our gagCEST sequence works in the context of degenerative IVD changes [5] [17] [20]. According to previous studies, the difference in GAG content between the NP and AF vanished in lumbar IVDs with protrusion or extrusion compared with discs with a normal appearance [16].

Our study has limitations. The main limitation of this study was the small sample size. For morphological (Pfirrmann classification) and biochemical MRI (gagCEST), we did not test intra- and interobserver agreement. The Pfirrmann classification has already demonstrated a good intra- and inter-reader reliability in prior studies [16]. For biochemical MRI, we used an automatic detection of GAG content of the NP and AF. For this reason, we believe that the missing reliability calculation is a minor limitation of both analyses. No gender, age, or BMI differentiation was considered in this study. Müller-Lutz and colleagues showed significantly lower GAG values in IVDs with increasing age and higher 
BMI [17]. These findings occur before morphological changes of IVDs could be revealed. This is a major limitation of our study considering the age difference between patients and control group and has to be taken into account in follow up studies. Moreover, for ethical reasons, we could not perform a histological correlation. We believe that the strength of this study is its focus on patients with radiculopathy. The results of our study show promise for evaluation of the effect in a larger population, applied to a patients' pain score or considering the difference between gender and age.

\section{Summary}

In summary, gagCEST of lumbar IVDs on a clinical 3T MRI system is a powerful, non-invasive tool without use of contrast medium to investigate early disc degeneration, predominantly concerning the NP. Biochemical imaging with gagCEST could provide an early biomarker for GAG loss in IVDs that may be on the way to develop degenerative changes like protrusion or extrusion, bony endplate alterations, formation of osteophytes, and consecutive spinal stenosis. Biochemical imaging of IVDs of patients with radiculopathy revealed that significantly lower GAG values compared with healthy controls, especially in the $\mathrm{NP}$, may indicate an association between pain and IVD degeneration.

\section{Conflicts of Interest}

The authors declare that they have no conflict of interest.

\section{References}

[1] Chou, R., Fu, R., Carrino, J.A. and Deyo, R.A. (2009) Imaging Strategies for Low-Back Pain: Systematic Review and Meta-Analysis. The Lancet, 373, 463-472. https://doi.org/10.1016/S0140-6736(09)60172-0

[2] Miles, D.E., Mitchell, E.A., Kapur, N., Beales, P.A. and Wilcox, RK. (2016) Peptide: Glycosaminoglycan Hybrid Hydrogels as an Injectable Intervention for Spinal Disc Degeneration. Journal of Materials Chemistry B, 4, 3225-3231. https://doi.org/10.1039/C6TB00121A

[3] Griffith, J.F., Wang, Y.X., Antonio, G.E., Choi, K.C., Yu, A., Ahuja, A.T., et al. (2007) Modified Pfirrmann Grading System for Lumbar Intervertebral Disc Degeneration. Spine, 32, e708-e712. https://doi.org/10.1097/BRS.0b013e31815a59a0

[4] An, H.S., Anderson, P.A., Haughton, V.M., Iatridis, J.C., Kang, J.D., Lotz, J.C., et al. (2004) Introduction: Disc Degeneration: Summary. Spine, 29, 2677-2678. https://doi.org/10.1097/01.brs.0000147573.88916.c6

[5] Urban, J.P. and Winlove, C.P. (2007) Pathophysiology of the Intervertebral Disc and the Challenges for MRI. Journal of Magnetic Resonance Imaging, 25, 419-432. https://doi.org/10.1002/jmri.20874

[6] Urban, J.P., McMullin, J.F. (1985) Swelling Pressure of the Inervertebral Disc: Influence of Proteoglycan and Collagen Contents. Biorheology, 22, 145-157. https://doi.org/10.3233/BIR-1985-22205

[7] Pfirrmann, C.W., Metzdorf, A., Zanetti, M., Hodler, J. and Boos, N. (2001) Magnetic Resonance Classification of Lumbar Intervertebral Disc Degeneration. Spine, 26, 1873-1878. https://doi.org/10.1097/00007632-200109010-00011 
[8] Rehnitz, C., Kupfer, J., Streich, N.A., Burkholder, I., Schmitt, B. Lauer, L., et al. (2014) Comparison of Biochemical Cartilage Imaging Techniques at 3 T MRI. Osteoarthritis and Cartilage, 22, 1732-1742. https://doi.org/10.1016/j.joca.2014.04.020

[9] Li, Y., Fredrickson, V. and Resnick, D.K. (2015) How Should We Grade Lumbar Disc Herniation and Nerve Root Compression? A Systematic Review. Clinical Orthopaedics and Related Research, 473, 1896-1902.

[10] Schleich, C., Miese, F., Müller, L. A., Boos, J., Aissa, J., Nasca, A., et al. (2016) Value of Delayed Gadolinium-Enhanced Magnetic Resonance Imaging of Cartilage for the Pre-Operative Assessment of Cervical Intervertebral Discs. The Journal of Orthopaedic Research, 35, 1824-1830.

[11] Vaga, S., Raimondi, M.T., Caiani, E.G., Costa, F., Giordano, C., Perona, F., et al. (2008) Quantitative Assessment of Intervertebral Disc Glycosaminoglycan Distribution by Gadolinium-Enhanced MRI in Orthopedic Patients. Magnetic Resonance in Medicine, 59, 85-95. https://doi.org/10.1002/mrm.21433

[12] Haneder, S., Ong, M.M., Budjan, J.M., Schmidt, R., Konstandin, S., Morelli, J.N., et al. (2014) ${ }^{23} \mathrm{Na}$-Magnetic Resonance Imaging of the Human Lumbar Vertebral Discs: In Vivo Measurements at 3.0 T in Healthy Volunteers and Patients with Low Back Pain. The Spine Journal, 14, 1343-1350.

https://doi.org/10.1016/j.spinee.2014.01.031

[13] Yoo, Y.H., Yoon, C.S., Eun, N.L., Hwang, M.J., Yoo, H., Peters, R.D., et al. (2016) Interobserver and Test-Retest Reproducibility of T1 $\rho$ and T2 Measurements of Lumbar Intervertebral Discs by 3T Magnetic Resonance Imaging. Korean Journal of Radiology, 17, 903-911. https://doi.org/10.3348/kjr.2016.17.6.903

[14] Ellingson, A.M., Mehta, H., Polly, D.W., Ellermann, J. and Nuckley, D.J. (2013) Disc Degeneration Assessed by Quantitative T2* (T2 Star) Correlated with Functional Lumbar Mechanics. Spine, 38, e1533-e1540. https://doi.org/10.1097/BRS.0b013e3182a59453

[15] Ling, W., Regatte, R.R., Navon, G. and Jerschow, A. (2008) Assessment of Glycosaminoglycan Concentration in Vivo by Chemical Exchange-Dependent Saturation Transfer (gagCEST). Proceedings of the National Academy of Sciences of the United States of America, 105, 2266-2270. https://doi.org/10.1073/pnas.0707666105

[16] Schleich, C., Müller, L.A., Eichner, M., Schmitt, B., Matuschke, F., Bittersohl, B., et al. (2016) Glycosaminoglycan Chemical Exchange Saturation Transfer of Lumbar Intervertebral Discs in Healthy Volunteers. Spine, 41, 146-152. https://doi.org/10.1097/BRS.0000000000001144

[17] Müller, L.A., Schleich, C., Schmitt, B., Antoch, G., Matuschke, F., Quentin, M., et al. (2015) Gender, BMI and T2 Dependencies of Glycosaminoglycan Chemical Exchange Saturation Transfer in Intervertebral Discs. Magnetic Resonance Imaging, 34, 271-275.

[18] Müller, L.A., Schleich, C., Pentang, G., Schmitt, B., Lanzman, R.S., Matuschke, F., et al. (2015) Age-Dependency of Glycosaminoglycan Content in Lumbar Discs: A 3t gagcEST Study. Journal of Magnetic Resonance Imaging, 42, 1517-1523. https://doi.org/10.1002/jmri.24945

[19] Saar, G., Zhang, B., Ling, W., Regatte, R.R., Navon, G. and Jerschow, A. (2012) Assessment of Glycosaminoglycan Concentration Changes in the Intervertebral Disc via Chemical Exchange Saturation Transfer. NMR in Biomedicine, 25, 255-261. https://doi.org/10.1002/nbm.1741

[20] Haneder, S., Apprich, S.R., Schmitt, B., Michaely, H.J., Schoenberg, S.O., Friedrich, K.M., et al. (2013) Assessment of Glycosaminoglycan Content in Intervertebral 
Discs Using Chemical Exchange Saturation Transfer at 3.0 Tesla: Preliminary Results in Patients with Low-Back Pain. European Radiology, 23, 861-868. https://doi.org/10.1007/s00330-012-2660-6

[21] Müller, L.A., Schleich, C., Schmitt, B., Topgöz, M., Pentang, G., Antoch, G., et al. (2014) Improvement of gagCEST Imaging in the Human Lumbar Intervertebral Disc by Motion Correction. Skeletal Radiology, 44, 505-511.

[22] Kim, M., Gillen, J., Landman, B.A., Zhou, J. and van Zijl, P.C. (2009) Water Saturation Shift Referencing (WASSR) for Chemical Exchange Saturation Transfer (CEST) Experiments. Magnetic Resonance in Medicine, 61, 1441-1450. https://doi.org/10.1002/mrm.21873

[23] Nachemson, A. (1975) Towards a Better Understanding of Low-Back Pain: A Review of the Mechanics of the Lumbar Disc. Rheumatology, 14, 129-143. https://doi.org/10.1093/rheumatology/14.3.129

[24] Videman, T., Battié, M.C., Gibbons, L.E., Maravilla, K., Manninen, H. and Kaprio, J. (2003) Associations between Back Pain History and Lumbar MRI Findings. Spine, 28, 582-588.

[25] Kraemer, J. (1995) Natural Course and Prognosis of Intervertebral Disc Diseases. Spine, 20, 635-639. https://doi.org/10.1097/00007632-199503150-00001 